\title{
Mode Competition in Relativistic Magnetrons and Injection Locking in KW Magnetrons*
}

\author{
V.B. Neculaes, R.M. Gilgenbach, M. Lopez, Y.Y. Lau, M. Jones, W. White, \\ P. Pengvanich, M.D. Johnston, T. Strickler, T.A. Spencer ${ }^{1}$, J. Luginsland ${ }^{2}$, \\ M. Haworth ${ }^{1}$, K. Cartwright ${ }^{1}$, P. Mardahl, ${ }^{1}$ T. Murphy, ${ }^{1}$ D. Price ${ }^{3}$ \\ Intense Energy Beam Interaction Laboratory \\ Nuclear Engineering and Radiological Sciences Dept. \\ University of Michigan, Ann Arbor, MI 48109 USA \\ ${ }^{1}$ Air Force Research Lab, Kirtland AFB, NM \\ ${ }^{2} \mathrm{SAIC}$ \\ ${ }^{3}$ Titan Corp.
}

\begin{abstract}
Both relativistic and nonrelativistic magnetrons are under experimental and theoretical investigation at U of M. Relativistic (Titan-6-vane) magnetron experiments (300-400 kV, 1-10 kA, 0.5 microsecond) investigate mode control with various output coupling geometries. Mode competition between the pi mode and the $2 / 3$ pi mode has been characterized for two-versus-three output extractors for comparison with particle in cell simulations. Phase measurements and timefrequency-analysis are performed for mode identification. Peak microwave output power on the order $0.5 \mathrm{GW}$ has been measured, assuming equal output from 3 waveguides.

Nonrelativistic $(4 \mathrm{kV},<1 \mathrm{~A}, \mathrm{~kW}$ microwave power) magnetron experiments are performed on commercial oven magnetrons for an in-depth investigation of crossed-field injection-locking and noise. Injection-locking is demonstrated by utilizing an oven magnetron as a reflection amplifier. Noise generation is explored as a function of injected signal and cathode conditions.
\end{abstract}

*Research supported by the AFOSR, AFRL, and the DUST (S\&T) under the Innovative Microwave Vacuum Electronics MURI Program Managed by the Air Force Office of Scientific Research, and Northrop Grumman Corp. 